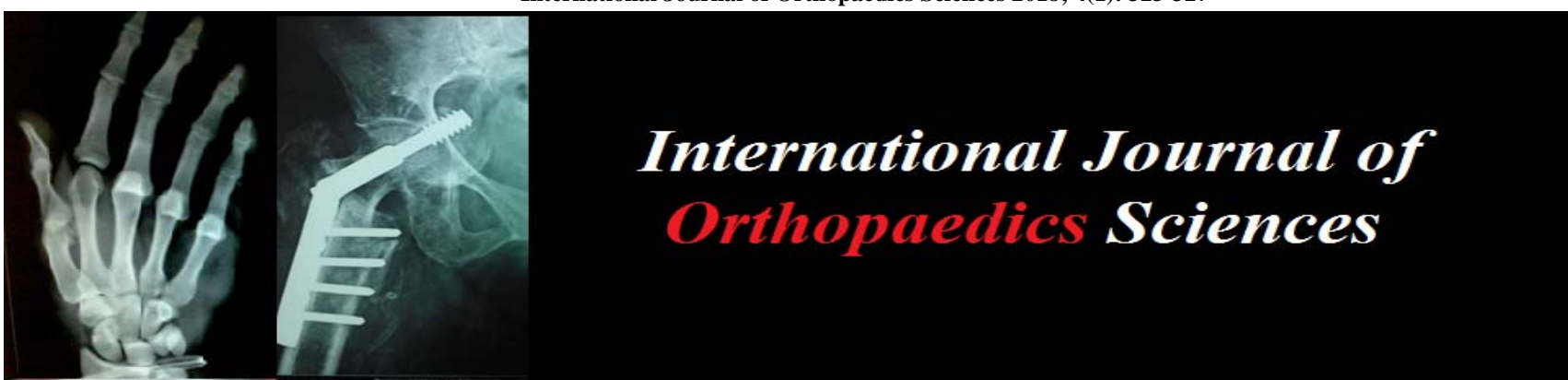

ISSN: $2395-1958$

IJOS 2018; 4(1): 323-327

(C) 2018 IJOS

www.orthopaper.com

Received: 12-11-2017

Accepted: 15-12-2017

Dr. Kanwarjit Singh Sandhu

Associate Professor, Department

of Orthopedics, Government

Medical College, Patiala,

Punjab, India

Dr. Ashok Kumar

Associate Professor, Department

of Microbiology, Government

Medical College, Patiala, Punjab, India

Dr. Ananta Singla

Junior Resident, Department of Microbiology, Government

Medical College, Patiala, Punjab, India

Ravinder K Banga

Senior Resident, Department of

Orthopedics, Government

Medical College, Patiala, Punjab, India

Dr. Karamdeep S Kahal Senior Resident, Department of Orthopedics, Government Medical College, Patiala, Punjab, India

Correspondence

Dr. Ashok Kumar

Associate Professor, Department of Microbiology, Government Medical College, Patiala, Punjab, India

\section{A study of prevalence of staphylococcus aureus and its antibiotic susceptibility pattern in 100 osteomyelitis patients in a tertiary care teaching hospital}

\author{
Dr. Kanwarjit Singh Sandhu, Dr. Ashok Kumar, Dr. Ananta Singla, \\ Ravinder K Banga, Dr. Karamdeep S Kahal
}

DOI: https://doi.org/10.22271/ortho.2018.v4.i1e.47

Abstract

Background: Osteomyelitis is an important cause of morbidity in patients with bone infections in developing countries like India. Gram positive bacteria especially Staphylococcus aureus, classically causes this desease, but Gram negative bacteria have grown in importance as causative agents. Widespread use of antibiotics has however altered the aetiological pattern of infections and antibiotic susceptibility pattern. Therefore, the treatment is becoming increasingly troublesome due to rise in drug resistant isolates.

Objective: This study was therefore undertaken to determine the bacteriological profile of osteomyelitis and also to ascertain the antibiotic susceptibility pattern of the isolates.

Materials and Methods: In the present study, 100 pus samples from osteomyelitis patients were taken and the specimens were inoculated onto Nutrient, blood and MacConkey agar plates. The plates were incubated at $37{ }^{\circ} \mathrm{C}$ for $18-24$ hours in an incubator. Culture isolates were identified by colony characteristics, Gram's staining, motility and biochemical reactions. Antibiotic sensitivity testing was done by Kirby Bauer disc diffusion method using CLSI guidelines. MRSA detection was done by cefoxitin disc diffusion method.

Results: The highest incidence of osteomyelitis was observed in the age group of 16-30 years (34\%) followed by $31-45$ years $(31 \%)$. Out of 100 cases, $76(76 \%)$ were males and $24(24 \%)$ were females with male to female ratio of 3.16:1. Trauma due to road side accidents $(45 \%)$ accounted for maximum no. of cases. The commonest organism isolated was Staphylococcus aureus - $32(35.56 \%)$ followed by Pseudomonas aeruginosa $-17(18.89 \%)$ as the second most common organism. Out of $32 \mathrm{Staph}$. aureus isolates, $13(41.93 \%)$ isolates were found to be MRSA and all the MRSA isolates were (100\%) resistant to ampicillin, amoxyclav and cefotaxime.

Conclusion : Our study will guide the clinicians in choosing appropriate antibiotics, which not only contribute to better treatment, but the judicious use of such antibiotics will also help in preventing emergence of resistance to the drugs, which are still sensitive.

Keywords: Osteomyelitis, Staphylococcus aureus, antibiotic susceptibility, Methicillin Resistant Staphylococcus aureus.

\section{Introduction}

Osteomyelitis or bone infection is an inflammation of bone caused by an infectious organism such as bacteria. However, fungi, parasites or viruses can also cause osteomyelitis ${ }^{[1]}$.

It affects both adults and children. Patients with certain conditions such as intravenous drug abusers, decubitus ulcers, surgery, trauma, immunosuppression, diabetes mellitus, smoking, malnutrition, malignancy and extremes of age are at increased risk of osteomyelitis [2]. Osteomyelitis can occur in a variety of bones in different areas of the body. The bones commonly involved in children are long bones such as femur, tibia, humerus and radius. In adults, it usually affects the vertebral column, in particular the lumbar spine, sacrum and the pelvis ${ }^{[1]}$.

Staphylococcus aureus is the most commonly isolated pathogen for both acute and chronic osteomyelitis in all age groups ${ }^{[2]}$. There has been increase in methicillin- resistant Staph. aureus (MRSA) in recent years due to rapid development of antimicrobial resistance and expression of virulence factors, regardless of the patient's immune status. 
Coagulase-negative staphylococci are often seen in association with foreign bodies, such as prosthetic joints ${ }^{[3]}$.

Also, Gram negative bacteria, such as Pseudomonas aeruginosa and Enterobacteriaceae have grown in importance as causative agents due to the rising number of high - energy traumas associated with open fractures ${ }^{[4]}$.

Widespread use of antibiotics has however altered the aetiological pattern of infections and antibiotic susceptibility pattern. Hence continuous monitoring of susceptibility pattern needs to be carried out so as to detect the true burden of antibiotic resistance among organisms and prevent their further emergence by judicious use of drugs ${ }^{[5]}$.

This study was therefore undertaken to determine the bacteriological profile of osteomyelitis and also to ascertain the antibiotic susceptibility pattern of the isolates that will help the clinician in deciding the treatment regime for the patients from the beginning of the treatment and avoid indiscriminate use of antibiotics.

\section{Materials and Methods}

In the present study, 100 pus samples from osteomyelitis patients attending the outdoor and indoor units of orthopaedics department, Rajindra hospital, Patiala were taken from July 2016 to Sep 2017. Pus was collected aseptically from the depth of the wound with a sterile syringe or swabs prior to administration of antimicrobial agents. The specimens were examined for colour, odour and consistency. Smears were made from pus and stained with Gram's staining to determine the morphology of organisms. For isolation of organisms, the specimens were inoculated onto Nutrient, blood and MacConkey agar plates. The plates were incubated at $37{ }^{\circ} \mathrm{C}$ for $18-24$ hours in an incubator. Culture isolates were identified by colony characteristics, Gram's staining, motility and biochemical reactions and any other special test for confirmation of particular organism.

Antibiotic sensitivity testing was done by Kirby Bauer disc diffusion method using CLSI guidelines ${ }^{[6]}$. The antibiotic discs were obtained from Hi - Media Laboratories Pvt. Ltd, Mumbai.

The various antibiotics used for Gram positive organisms were:- ampicillin, gentamicin, amikacin, amoxyclav, ciprofloxacin, cotrimoxazole, cefoxitin, cefotaxime, erythromycin, clindamycin, linezolid and vancomycin. The antibiotics used for Gram negative organisms were:ampicillin, gentamicin, amikacin, amoxyclav, ciprofloxacin, cotrimoxazole, cefotaxime, ceftazidime, ceftazidime-clav, piperacillin/tazobactam and imipenem.

CLSI recommends cefoxitin disc diffusion method for MRSA detection. It was performed by using cefoxitin disc on Mueller-Hinton medium and plates were incubated at 33-35 ${ }^{\circ} \mathrm{C}$ for $16-18$ hours and zone diameter was measured in the presence of reflected light.

\begin{tabular}{|c|c|c|}
\hline \multicolumn{3}{|c|}{ Interpretative Criteria for Cefoxitin Disc Diffusion Test - in mm } \\
\hline & Susceptible & Resistant \\
\hline S. aureus & $\geq 22 \mathrm{~mm}$ & $\leq 21 \mathrm{~mm}$ \\
\hline
\end{tabular}

\section{Results}

The highest incidence of osteomyelitis was observed in the age group of $16-30$ years $(34 \%)$ followed by $31-45$ years $(31 \%),>46$ years $(24 \%)$ and $0-15$ years $(11 \%)$. The age range was found to be 5-75 years while the median age was observed as 34 years. Out of 100 cases, $76(76 \%)$ were males and $24(24 \%)$ were females with male to female ratio of 3.16:1.

The predisposing factors for osteomyelitis are listed in table 1. Trauma due to road side accidents (45\%) accounted for maximum no. of cases. Other factors responsible were postoperative infections (21\%), orthopaedic implants $(17 \%)$, haematogenous (11\%), diabetes mellitus (4\%) and others $(2 \%)$

Table 1: Predisposing factors for osteomyelitis

\begin{tabular}{|c|c|c|c|}
\hline $\begin{array}{c}\text { Sr. } \\
\text { No. }\end{array}$ & Pre disposing factors & $\begin{array}{c}\text { No. of } \\
\text { isolates }\end{array}$ & Percentage \\
\hline 1 & $\begin{array}{c}\text { Trauma due to road side } \\
\text { accidents }\end{array}$ & 45 & $45 \%$ \\
\hline 2 & Post-operative infections & 21 & $21 \%$ \\
\hline 3 & Orthopaedic implants & 17 & $17 \%$ \\
\hline 4 & Haematogenous & 11 & $11 \%$ \\
\hline 5 & Diabetes mellitus & 4 & $4 \%$ \\
\hline 6 & Others & 2 & $2 \%$ \\
\hline \multicolumn{2}{r|}{} & 100 & $100 \%$ \\
\hline
\end{tabular}

Table 2 shows the order of involvement of various bones. Tibia was the most commonly involved bone in $36 \%$ cases, followed by femur (32\%), bones of foot $(9 \%)$, humerus $(6 \%)$, radius $(5 \%)$, bones of hand $(4 \%)$, fibula and ulna $(3 \%$ each) and clavicle $(2 \%)$.

Table 2: Bones involved in osteomyelitis

\begin{tabular}{|c|c|c|c|}
\hline Sr. No. & Bones involved & No. of isolates & Percentage \\
\hline 1 & Tibia & 36 & $36 \%$ \\
\hline 2 & Femur & 32 & $32 \%$ \\
\hline 3 & Bones of foot & 9 & $9 \%$ \\
\hline 4 & Humerus & 6 & $6 \%$ \\
\hline 5 & Radius & 5 & $5 \%$ \\
\hline 6 & Bones of hand & 4 & $4 \%$ \\
\hline 7 & Fibula & 3 & $3 \%$ \\
\hline 8 & Ulna & 3 & $3 \%$ \\
\hline 9 & Clavicle & 2 & $2 \%$ \\
\hline \multicolumn{2}{|r|}{ Total } & 100 & $100 \%$ \\
\hline
\end{tabular}

In a total of 100 cultures put up, growth of organisms was obtained in $84(84 \%)$ cases and $16(16 \%)$ cases were sterile. Out of 84 positive cultures, single organism was obtained in $78(92.86 \%)$ cases and two organisms were isolated in 6 (7.14\%) cases accounting for a total of 90 isolates (Figure 1).

The commonest organism isolated was Staphylococcus aureus - $32(35.56 \%)$ followed by Pseudomonas aeruginosa -17 $(18.89 \%)$ as the second most common organism. (Figure 2)

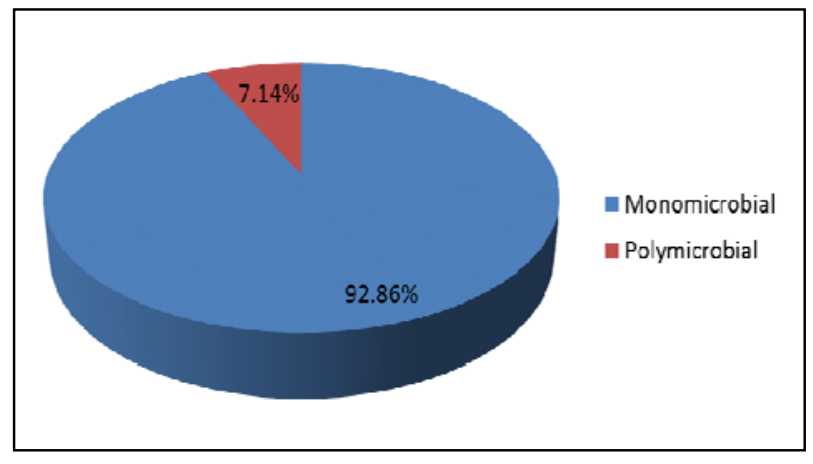

Fig 1: Type of Isolate found in positive culture cases 


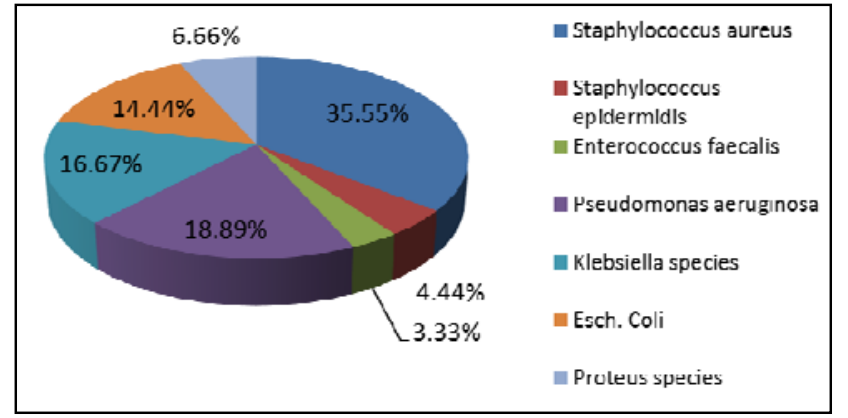

Fig 2: Percentage of various pathogens in positive culture cases

Table 3 shows the antibiotic susceptibility pattern of Gram positive isolates. Staph.aureus showed $100 \%$ sensitivity to vancomycin followed by linezolid $(96.87 \%)$, clindamycin $(78.12 \%)$ and amikacin (71.87\%). Staph. epidermidis was found to be $100 \%$ sensitive to vancomycin followed by $75 \%$ sensitivity to each of linezolid and amikacin. E. faecalis showed resistance to all other drugs except vancomycin, linezolid and amikacin. Table 4 shows the antibiotic susceptibility pattern of Gram negative isolates.

Table 3: Antibiotic susceptibility pattern of Gram positive isolates

\begin{tabular}{|c|c|c|c|c|}
\hline Sr. No. & Antibiotic & $\begin{array}{c}\text { Staph. aureus } \\
(\mathbf{n}=\mathbf{3 2})\end{array}$ & $\begin{array}{c}\text { Staph. epidermidis } \\
(\mathbf{n}=\mathbf{4})\end{array}$ & $\begin{array}{c}\text { E. faecalis } \\
(\mathbf{n = 3})\end{array}$ \\
\hline 1 & Ampicillin & $5(15.62 \%)$ & $1(25 \%)$ & $0(0 \%)$ \\
\hline 2 & Gentamicin & $16(50 \%)$ & $1(25 \%)$ & $0(0 \%)$ \\
\hline 3 & Amikacin & $23(71.87 \%)$ & $3(75 \%)$ & $1(33.33 \%)$ \\
\hline 4 & Amoxyclav & $11(34.37 \%)$ & $2(50 \%)$ & $0(0 \%)$ \\
\hline 5 & Ciprofloxacin & $10(31.25 \%)$ & $1(25 \%)$ & $0(0 \%)$ \\
\hline 6 & Cotrimoxazole & $16(50 \%)$ & $2(50 \%)$ & $0(0 \%)$ \\
\hline 7 & Cefoxitin & $19(59.37 \%)$ & $2(50 \%)$ & $0(0 \%)$ \\
\hline 8 & Cefotaxime & $13(40.62 \%)$ & $3(75 \%)$ & $0(0 \%)$ \\
\hline 9 & Erythromycin & $15(46.87 \%)$ & $2(50 \%)$ & $0(0 \%)$ \\
\hline 10 & Clindamycin & $25(78.12 \%)$ & $2(50 \%)$ & $0(0 \%)$ \\
\hline 11 & Linezolid & $31(96.87 \%)$ & $3(75 \%)$ & $3(100 \%)$ \\
\hline 12 & Vancomycin & $32(100 \%)$ & $4(100 \%)$ & $3(100 \%)$ \\
\hline
\end{tabular}

Table 4: Antibiotic susceptibility pattern of Gram negative isolates

\begin{tabular}{|c|c|c|c|c|c|c|c|c|c|}
\hline Sr. No. & Antibiotic & \multicolumn{2}{|c|}{$\begin{array}{c}\text { P. aeruginosa } \\
(\mathbf{n = 1 7 )}\end{array}$} & \multicolumn{2}{|c|}{$\begin{array}{c}\text { Klebsiella spp. } \\
\text { (n=15) }\end{array}$} & \multicolumn{2}{|c|}{$\begin{array}{c}\text { Esch. coli } \\
\text { (n=13) }\end{array}$} & \multicolumn{2}{c|}{$\begin{array}{c}\text { Proteus spp. } \\
\text { (n=6) }\end{array}$} \\
\hline 1 & Ampicillin & 0 & $0 \%$ & 0 & $0 \%$ & 2 & $15.38 \%$ & 0 & $0 \%$ \\
\hline 2 & Gentamicin & 4 & $23.53 \%$ & 11 & $73.33 \%$ & 4 & $30.76 \%$ & 2 & $33.33 \%$ \\
\hline 3 & Amikacin & 9 & $52.94 \%$ & 12 & $80 \%$ & 11 & $84.61 \%$ & 4 & $66.66 \%$ \\
\hline 4 & Amoxyclav & 7 & $41.18 \%$ & 0 & $0 \%$ & 4 & $30.76 \%$ & 0 & $0 \%$ \\
\hline 5 & Ciprofloxacin & 7 & $41.18 \%$ & 7 & $46.66 \%$ & 8 & $61.54 \%$ & 0 & $0 \%$ \\
\hline 6 & Cotrimoxazole & 4 & $23.53 \%$ & 5 & $33.33 \%$ & 5 & $38.46 \%$ & 0 & $0 \%$ \\
\hline 7 & Cefotaxime & 4 & $23.53 \%$ & 7 & $46.66 \%$ & 4 & $30.76 \%$ & 0 & $0 \%$ \\
\hline 8 & Ceftazidime & 3 & $17.64 \%$ & 2 & $13.33 \%$ & 5 & $38.46 \%$ & 0 & $0 \%$ \\
\hline 9 & Ceftazidime-clav & 4 & $23.53 \%$ & 3 & $20 \%$ & 6 & $46.15 \%$ & 0 & $0 \%$ \\
\hline 10 & Piperacillin-tazobactam & 9 & $52.94 \%$ & 4 & $26.66 \%$ & 12 & $92.30 \%$ & 5 & $83.33 \%$ \\
\hline 11 & Imipenem & 12 & $70.58 \%$ & 12 & $80 \%$ & 13 & $100 \%$ & 5 & $83.33 \%$ \\
\hline
\end{tabular}

Out of 32 Staph. aureus isolates, 13 (41.93\%) isolates were found to be MRSA and 19 (59.37\%) isolates obtained were MSSA. The antibiotic sensitivity pattern of MRSA was observed as follows - vancomycin (100\%), linezolid (100\%), clindamycin (61.54\%), amikacin (61.54\%), cotrimoxazole (38.46\%), erythromycin $(30.77 \%)$, gentamicin $(7.70 \%)$, ciprofloxacin (7.69\%). All the MRSA isolates were (100\%) resistant to ampicillin, amoxyclav and cefotaxime.

Table 5: Antibiotic susceptibility pattern of MRSA and MSSA isolates

\begin{tabular}{|c|c|c|c|c|c|c|c|c|}
\hline \multirow{2}{*}{ Sr. No. } & \multirow{2}{*}{ Antibiotic } & & \multicolumn{2}{|c|}{ MRSA (n=13) } & \multicolumn{2}{|c|}{ MSSA (n=19) } & \multirow{2}{*}{$\begin{array}{c}\text { Chi Sqaure } \\
X^{2} \\
\end{array}$} & \multirow{2}{*}{$p$ value } \\
\hline & & & Number & Percent & Number & Per cent & & \\
\hline \multirow[b]{2}{*}{1} & \multirow{2}{*}{ Ampicillin } & Sensitive & 0 & $0 \%$ & 5 & $26.31 \%$ & \multirow[b]{2}{*}{4.055} & \multirow[b]{2}{*}{$0.044(\mathrm{~S})$} \\
\hline & & Resistant & 13 & $100 \%$ & 14 & $73.69 \%$ & & \\
\hline \multirow{2}{*}{2} & \multirow{2}{*}{ Gentamicin } & Sensitive & 2 & $15.38 \%$ & 14 & $73.69 \%$ & \multirow{2}{*}{10.494} & \multirow{2}{*}{$0.001(\mathrm{~S})$} \\
\hline & & Resistant & 11 & $84.62 \%$ & 5 & $26.31 \%$ & & \\
\hline \multirow{2}{*}{3} & \multirow{2}{*}{ Amikacin } & Sensitive & 8 & $61.54 \%$ & 15 & $78.95 \%$ & \multirow{2}{*}{1.157} & \multirow{2}{*}{$0.282(\mathrm{NS})$} \\
\hline & & Resistant & 5 & $38.46 \%$ & 4 & $21.05 \%$ & & \\
\hline \multirow{2}{*}{4} & \multirow{2}{*}{ Amoxyclav } & Sensitive & 0 & $0 \%$ & 11 & $57.90 \%$ & \multirow{2}{*}{11.469} & \multirow{2}{*}{$0.001(\mathrm{~S})$} \\
\hline & & Resistant & 13 & $100 \%$ & 8 & $42.10 \%$ & & \\
\hline \multirow{2}{*}{5} & \multirow{2}{*}{ Ciprofloxacin } & Sensitive & 1 & $7.70 \%$ & 9 & $47.37 \%$ & \multirow[b]{2}{*}{5.656} & \multirow{2}{*}{$0.017(\mathrm{~S})$} \\
\hline & & Resistant & 12 & $92.30 \%$ & 10 & $52.63 \%$ & & \\
\hline \multirow{2}{*}{6} & \multirow{2}{*}{ Cotrimoxazole } & Sensitive & 5 & $38.46 \%$ & 11 & $57.90 \%$ & \multirow{2}{*}{1.166} & \multirow{2}{*}{$0.280(\mathrm{NS})$} \\
\hline & & Resistant & 8 & $61.54 \%$ & 8 & $42.10 \%$ & & \\
\hline \multirow{2}{*}{7} & \multirow{2}{*}{ Cefoxitin } & Sensitive & 0 & $0 \%$ & 19 & $100 \%$ & & \\
\hline & & Resistant & 13 & $100 \%$ & 0 & $0 \%$ & 32.00 & $<0.001(\mathrm{~S})$ \\
\hline & & Sensitive & 0 & $0 \%$ & 13 & $68.42 \%$ & & \\
\hline 8 & Cefotaxime & Resistant & 13 & $100 \%$ & 6 & $31.58 \%$ & 14.981 & $11(\mathrm{~S})$ \\
\hline 0 & & Sensitive & 4 & $30.77 \%$ & 11 & $57.90 \%$ & 2281 & 0.131 \\
\hline 9 & Erythromycin & Resistant & 9 & $69.23 \%$ & 8 & $42.10 \%$ & 51 & (NS) \\
\hline & & Sensitive & 8 & $61.54 \%$ & 17 & $89.47 \%$ & & 0.060 \\
\hline 10 & Clindamycin & Resistant & 5 & $38.46 \%$ & 2 & $10.53 \%$ & 3.525 & (NS) \\
\hline & & Sensitive & 13 & $100 \%$ & 18 & $94.74 \%$ & 070 & 0.401 \\
\hline 11 & Lir & Resistant & 0 & $0 \%$ & 1 & $5.26 \%$ & 0.706 & (NS) \\
\hline 12 & & Sensitive & 13 & $100 \%$ & 19 & $100 \%$ & & \\
\hline 12 & Vancomycin & Resistant & 0 & $0 \%$ & 0 & $0 \%$ & & \\
\hline
\end{tabular}

NS (Not Significant) $p>0.05$; S (Significant) $p<0.05$ at $\mathrm{df}=1$, NA $=$ not applicable 


\section{Discussion}

The highest incidence of osteomyelitis was observed in the age group of $16-30$ years (34\%) followed by $31-45$ years $(31 \%),>46$ years $(24 \%)$ and $0-15$ years $(11 \%)$ Similar observations were made in a study by Kaur $\mathrm{J}$ et al. ${ }^{[5]}$, who showed that occurence of osteomyelitis was higher in 16-30 $(36 \%)$ and $31-45(34 \%)$ age groups than in children and elderly age group and by Qureshi $\mathrm{M}$ et al. ${ }^{[7]}$ who showed that maximum cases of osteomyelitis were found in age group of $15-30$ years $(32.7 \%)$ followed by $31-50$ age group (31.5\%). In our study, osteomyelitis cases were more common in males $(76 \%)$ as compared to females $(24 \%)$ with a male: female ratio of 3.16:1. Similar results were reported by Kumar A et al. ${ }^{[8]}$, Jiang $\mathrm{N}$ et al. ${ }^{[9]}$ and Singh A et al. ${ }^{[10]}$ who showed male: female ratio to be $2.72: 1,3.53: 1$ and $2.7: 1$ respectively. In the current study, the most common predisposing factor leading to osteomyelitis was trauma due to road side accidents accounting for $45 \%$ cases followed by post-operative infections (21\%), orthopaedic implants (17\%), haematogenous $(11 \%)$, diabetes mellitus $(4 \%)$ and others. This is in concordance with a study by Wadekar MD et al. ${ }^{[11]}$ who concluded trauma (44\%) as the commonest predisposing factor followed by post-operative infections (23\%) and orthopaedic implants (23\%) and also similar to the findings of Khatoon $\mathrm{R}$ et al. ${ }^{[12]}$ according to whom the most common predisposing factor was trauma leading to open fractures $(45.6 \%)$

In the present study, it was found that tibia was the commonest bone affected by the osteomyelitis (36\%), followed by femur $(32 \%)$. However, our study differs from study of Wadekar MD et al. [11] which showed highest incidence of osteomyelitis in femur (48\%) followed by tibia $(23 \%)$.

Out of 100 pus samples processed in the present study, $84 \%$ were culture positive and $16 \%$ were culture negative This finding is in concordance with studies by Veerana HD et al. ${ }^{[13]}$ and Suguneshwari G et al. ${ }^{[14] .}$

Out of 84 positive cultures, single organism was isolated in $78(92.86 \%)$ cases and two organisms were isolated in 6 (7.14\%) cases. These results are consistent with a study by Salim $\mathrm{N}$ et al. ${ }^{[15]}$ which showed $87.27 \%$ monomicrobial growth and $12.72 \%$ polymicrobial growth. A similar study by Shah FA et al. ${ }^{[16]}$ from Pakistan also reported $92 \%$ growth as monomicrobial and $8 \%$ as polymicrobial.

In the present study, among the Gram positive organisms, Staph.aureus was isolated from maximum no. of patients (35.55\%) followed by Staph. epidermidis (4.44\%) and Enterococcus faecalis (3.33\%) which is similar to the studies of Kaur J et al. ${ }^{[5]}$ who isolated Staph. aureus in maximum no. of cases - 43\% followed by Staph. epidermidis in $4 \%$ cases. Sheehy $\mathrm{SH}$ et al. ${ }^{[17]}$ in his prospective study on 166 patients from Oxford, UK also reported Staph. aureus as the most common isolate (32\%) amongst a wide range of organisms.

Among the Gram negative organisms, the most commonly isolated pathogen was Pseudomonas aeruginosa (18.89\%) followed by Klebsiella species (16.67\%), Esch. coli (14.44\%) and Proteus spp. (6.66\%). However, our study differs from observations of Kumar A et al. ${ }^{[8]}$ who reported maximum incidence in E. coli (12.1\%) and Gopi A et al. ${ }^{[18]}$ who isolated Klebsiellapneumoniae in maximum no. of cases as $11.4 \%$.

In the present study, Staph.aureus showed maximum sensitivity to vancomycin $(100 \%)$ followed by linezolid (96.87\%), clindamycin (78.12\%) and amikacin (71.87\%). Our findings are supported by Gopi A et al. ${ }^{[18]}$ who reported $100 \%$ sensitivity to both vancomycin and linezolid followed by high degree of sensitivity to clindamycin (94\%). Chakravarty $\mathrm{S}$ et al. ${ }^{[19]}$ also reported similar sensitivity pattern with sensitivity to vancomycin, linezolid and amikacin as $100 \%, 95.7 \%$ and $78.3 \%$ respectively.

Our study results showed that out of 39 strains of Staph. aureus, $41.93 \%$ strains were MRSA and MSSA accounted for $59.37 \%$ of the cases. Our study correlates well to the study of Salim N et al. ${ }^{[15]}$ who reported incidence of MRSA and MSSA being 38.88\% and $61.11 \%$ respectively.

In the present study, majority of the MRSA strains were resistant to commonly used antibiotics but showed 100\% sensitivity to both vancomycin and linezolid followed by sensitivity to clindamycin and amikacin in equal proportion (61.54\% each). These findings are comparable to the study of Wadekar MD et al. ${ }^{[11]}$ who also reported $100 \%$ sensitivity to both vancomycin and linezolid followed by $78.5 \%$ sensitivity to amikacin. Ali $\mathrm{M}$ et al. ${ }^{[20]}$ in his study reported $100 \%$ sensitivity to vancomycin and $75 \%$ sensitivity to linezolid and high degree of resistance to commonly used antibiotics like ciprofloxacin and cefotaxime.

It is quite clear from the previous studies and the present study that MRSA strains are becoming alarming because of their increased resistance towards antibiotics - like amikacin and gentamicin, that leaves the clinicians with limited choice to use appropriate drug for the treatment of osteomyelitis.

\section{Conclusion}

Osteomyelitis is even today one of the most challenging bone and joint infections. Gram positive bacteria especially Staphylococcus aureus, classically causes this desease, but Gram negative bacteria have grown in importance as causative agents. Gram negative bacilli and methicilin resistant Staph. aureus has been found to be quite high among the organisms isolated from osteomyelitis patients.

Emerging multidrug resistant strains is of major concern as they pose challenge in the treatment of osteomyelitis. Multidrug resistance among MRSA strains has serious implications as far as treatment of MRSA infections is concerned.

This study highlights the importance of culture-directed antibiotic therapy for osteomyelitis and thus helps the orthopaedician in choosing appropriate antibiotics which not only contribute to better treatment but their judicious use will also help in preventing emergence of resistance to the antibiotics which are still sensitive.

\section{References}

1. Helgeson Kevin. Infectious diseases of the musculoskeletal system.In: Goodman CC, Fuller KS,editors. Pathology: implications for the physical therapist. 4th ed. St Louis, MO: Saunders. 2015, 1234-6.

2. Eid AJ, Berbari EF. Osteomyelitis: a review of Pathophysiology, diagnostic modalities and therapeuticoptions. J Med Liban. 2012; 60:51-60.

3. Chihara S, Segreti J. Osteomyelitis. Dis Mon. 2010; 56(1):5-31.

4. de Carvalho VC, de Oliveira PRD, Dal - Paz K, de Paula AP, Felix CSF, Lima LLM. Gram negative osteomyelitis: clinical and microbiological profile. Braz J Infect Dis. 2012; 16(1):63-7.

5. Kaur J, Gulati VL, Aggarwal A, Gupta V. Bacteriological profile of osteomyelitis with special reference to Staphylococcus aureus. IndJ Pract Doctor. 2008; 4(6):1- 
9.

6. Clinical and Laboratory Standards Institute. Performance Standards for Antimicrobial Susceptibility Testing; Twenty - Fifth Informational Supplement document. M100-S25. Wayne, PA: Clinical and Laboratory Standards Institute, 2015, 1-236.

7. Qureshi M, Chaudry S, Haroon S. Bacterial aetiology of bone lesions in a tertiary care hospital. Biomedica. 2009; 25:180-3.

8. Kumar A, Singh U. Bacteriological pattern of chronic osteomyelitis- A retrospective study. J Evid based Med Healthc. 2017; 4(39):2387-90.

9. Jiang N, Ma YF, Jiang Y, Zhao X. Clinical characteristics and treatment of extremity chronic Osteomyelitis in Southern China: A retrospective analysis of 394 consecutive patients. Medicine (Baltimore). 2015; 94(42):e1874.

10. Singh A, Biswas PP, Sen A. Bacteriological profile of osteomyelitis cases with special reference to antibiotic susceptibility pattern of isolates in a tertiary care hospital of eastern India. J Evolution Med Dent Sci. 2016; 5(53):3496-501.

11. Wadekar MD, Naganath M, Venkatesha D. Detection of ESBL, MBL and MRSA among isolates of chronic osteomyelitis and their antibiogram. Int $\mathrm{J}$ CurrMicrobiolAppl Sci. 2015; 4(10):289-95.

12. Khatoon R, Khan SA, Jahan N. Antibiotic resistance pattern among aerobic bacterial isolates from osteomyelitis cases attending a tertiary care hospital of North India with special reference to ESBI, AmpC, MBL and MRSA production. Int $\mathrm{J}$ Res Med Sci. 2017; 5(2):482-90.

13. Veeranna HD, Arif M, Azeem A. A Retrospective Analysis of Efficacy of Non - Surgical treatment for Diabetic Chronic Osteomyelitis. J Evolution Med Dent Sci. 2014; 3:8313-6.

14. Suguneswari G, Singh AH, Basu R. Bacteriological profile of Osteomyelitis in a tertiary care hospital at Vishakhapatnam, Andhra Pradesh. Int J Cur Res and Rev. 2013; 5(20):52-8.

15. Salim N, Lancy J. Bacteriological profile of posttraumatic osteomyelitis in a tertiary care centre. Int $\mathrm{J}$ CurrMicrobiol App Sci. 2017; 6(1):367-72.

16. Shah FA, Khan Z, Ali W, Durrani ZA. Chronic osteomyelitis; microbiology of long bones in a tertiary care hospital. Professional Med J. 2012; 19(5):719-22.

17. Sheehy SH, Atkins BA, Bejon P. The microbiology of chronic osteomyelitis: prevalence of resistance to common empirical anti-microbial regimens. J Infect. 2010; 60(5):338-43.

18. Gopi A, Khair SMU, Kottileveetil HT. A clinicomicrobiological study of osteomyelitis in a tertiary care hospital in Karnataka. J Evolution Med Dent Sci. 2016; $5(1): 15-8$.

19. Chakravarty S, Kataki M, Pradhan U, Yangzom T. Chronic osteomyelitis: a bacteriological Study with special reference to Staphylococcus aureus. J evid based Med Healthc. 2015; 2(2):89-96.

20. Ali M, Kumari R. Evaluation of Bacteriological Profile of Chronic Osteomyelitis in a Tertiary Care Hospital. Int J Scientific Research. 2014; 3(11):383-5. 